\title{
Entanglement in SU(2)-invariant quantum spin systems
}

\author{
John Schliemann \\ Department of Physics and Astronomy, University of Basel, CH-4056 Basel, Switzerland
}

(Received 19 December 2002; published 11 July 2003)

\begin{abstract}
We analyze the entanglement of SU(2)-invariant density matrices of two spins $\vec{S}_{1}, \vec{S}_{2}$ using the PeresHorodecki criterion. Such density matrices arise from thermal equilibrium states of isotropic-spin systems. The partial transpose of such a state has the same multiplet structure and degeneracies as the original matrix with the eigenvalue of largest multiplicity being non-negative. The case $S_{1}=S, S_{2}=1 / 2$ can be solved completely and is discussed in detail with respect to isotropic Heisenberg spin models. Moreover, in this case the PeresHorodecki criterion turns out to be a sufficient condition for nonseparability. We also characterize SU(2)invariant states of two spins of length 1 .
\end{abstract}

DOI: 10.1103/PhysRevA.68.012309

PACS number(s): 03.67.Mn, 75.10.Jm, 75.10.Pq

\section{INTRODUCTION}

Entanglement is one of the most intriguing properties of quantum physics $[1,2]$ and the key ingredient of the emerging field of quantum information theory and processing [3]. Recently, substantial interest has developed concerning the question of quantum entanglement in equilibrium states of quantum spin systems [7-22] as often studied in condensedmatter physics and statistical mechanics. More specifically, a typical question arises: Tracing out from a many-body system all degrees of freedom except for, say, two spins, is this reduced density matrix separable or not?

In the present work, we study SU(2)-invariant density matrices of two spins. These states are defined to be invariant under all uniform rotations $U_{1} \otimes U_{2}$ of both spins $\vec{S}_{1}$ and $\vec{S}_{2}$, where $U_{a}=\exp \left(i \vec{\eta} \cdot \vec{S}_{a}\right), a \in\{1,2\}$, are transformations corresponding to the same set of real parameters $\vec{\eta}$ in the representation of SU(2), appropriate for the spin lengths $S_{1}$ and $S_{2}$ $(\hbar=1)$. In other words, such states, $\rho$, commute with all the components of the total spin $\vec{J}=\vec{S}_{1}+\vec{S}_{2}$. SU(2)-invariant density matrices arise from thermal equilibrium states of spin systems with an rotationally invariant Hamiltonian by tracing out all degrees of freedom, except those two spins [23]. Our results generalize the previous work on such systems $[7,8,13,14]$ to the case of higher spins, and we discuss our findings with respect to generic antiferromagnetic or ferrimagnetic Heisenberg spin-lattice models. These considerations lead to the natural conclusion that pairwise quantum entanglement in equilibrium states is the strongest in systems with small spin length and low spatial dimension. Moreover, an inseparable, equilibrium, reduced, two-spin state can usually only be achieved for neighboring spins, but not for more distant lattice sites. Viewed in this manner, equilibrium states of such systems do not appear to be a particularly strong source of pairwise quantum entanglement.

To investigate the separability of this type of density matrices in the case of higher spins we shall make use of the Peres-Horodecki criterion $[24,25]$. This criterion states that a separable density matrix has necessarily a positive partial transpose [24]. Moreover, a positive partial transpose is also sufficient [25] for the separability of a given density matrix in the case of two qubits, and in the case of a qubit and a qutrit (i.e., the case of two spins of length $1 / 2$, and of a spin $1 / 2$ and a spin 1, respectively). For larger dimensions of the parties (spins) involved, nonseparable states with positive partial transpose exist [26]. To apply the Peres-Horodecki criterion one can perform the partial transposition with respect to either subsystem (spin), since both resulting matrices have the same spectrum. For definiteness, we will consider in the following the partial transpose $\rho^{T_{2}}$ with respect to $\vec{S}_{2}$.

$\mathrm{SU}(2)$-invariant two-spin states were mentioned briefly already by Vollbrecht and Werner [27], where it was pointed out that the case $S_{1}=S_{2}=1$ corresponds to states invariant under $O \otimes O$ with $O$ being an $\mathrm{O}(3)$ rotation. In this work, we will also give explicit criteria in terms of spin correlators for such states to have a positive partial transpose [28].

\section{SU(2)-INVARIANT STATES AND THEIR PARTIAL TRANSPOSE}

Let us start with some general considerations. Since an $\mathrm{SU}(2)$-invariant state commutes with all components of $\vec{J}$, it acts, according to Schur's Lemma, as a scalar on each irreducible representation (multiplet) of $\vec{J}$. Therefore, $\rho$ has the general from

$$
\rho=\sum_{J=\left|S_{1}-S_{2}\right|}^{S_{1}+S_{2}} \frac{A(J)}{2 J+1} \sum_{J^{z}=-J}^{J}\left|J, J^{z}\right\rangle_{00}\left\langle J, J^{z}\right|,
$$

where the constants $A(J)$ fulfill $A(J) \geqslant 0, \Sigma_{J} A(J)=1$. Here, $\left|J, J^{z}\right\rangle_{0}$ denotes a state of total spin $J$ and $z$ component $J^{z}$.

Now let $\mathcal{O}$ be a general operator acting on a bipartite system. If $\mathcal{O}$ is transformed by $U_{1} \otimes U_{2}, \mathcal{O}^{T_{2}}$ transforms covariantly under $U_{1} \otimes U_{2}^{*}$,

$$
\left[\left(U_{1} \otimes U_{2}\right) \mathcal{O}\left(U_{1}^{+} \otimes U_{2}^{+}\right)\right]^{T_{2}}=\left(U_{1} \otimes U_{2}^{*}\right) \mathcal{O}^{T_{2}}\left[U_{1}^{+} \otimes\left(U_{2}^{*}\right)^{+}\right]
$$

Here, $U_{1}, U_{2}$ are general unitary transformations acting on the subsystems and do not necessarily represent SU(2) transformations. Relation (2) can be derived readily by writing $\mathcal{O}$ 
in the form $\mathcal{O}_{i_{1} i_{2}, j_{1} j_{2}}$, where the subscripts at the indices refer to the different subsystems $[27,29]$. In particular, it follows that if $\mathcal{O}$ is invariant under $U_{1} \otimes U_{2}, \mathcal{O}^{T_{2}}$ is invariant under $U_{1} \otimes U_{2}^{*}$.

Now let $U_{1}, U_{2}$ represent again $\mathrm{SU}(2)$ transformations. In the standard representations of their generators the $x$ and $z$ components are given by real matrices, while the matrices for the $y$ components are imaginary. Thus, a complex conjugation of $U_{2}=\exp \left(i \vec{\eta} \cdot \vec{S}_{2}\right)$ is equivalent to changing the sign of $S_{2}^{x}, S_{2}^{z}$. Therefore, $\rho^{T_{2}}$ commutes with the operators $\vec{K}$ defined by $K^{x}=S_{1}^{x}-S_{2}^{x}, K^{y}=S_{1}^{y}+S_{2}^{y}, K^{z}=S_{1}^{z}-S_{2}^{z}$, and these operators also furnish a representation of $\mathrm{su}(2)$, $\left[K^{\alpha}, K^{\beta}\right]=i \varepsilon^{\alpha \beta \gamma} K^{\gamma}$ (using standard notation). In the basis of tensor product states $\left|S_{1}^{z}, S_{2}^{z}\right\rangle$ of $S_{1}^{z}, S_{2}^{z}, \rho^{T_{2}}$ is block diagonal with respect given to the values of $K^{z}$. In particular, $\left| \pm S_{1}, \mp S_{2}\right\rangle$ are eigenstates of $\rho^{T_{2}}$ with the degenerate eigenvalue $\left\langle \pm S_{1}, \mp S_{2}|\rho| \pm S_{1}, \mp S_{2}\right\rangle \geqslant 0$. Now it follows from the elementary representation theory that $\rho^{T_{2}}$ has actually an SU(2) multiplet structure with respect to the operators $\vec{K}$. The multiplets are labeled by the value of $\vec{K}^{2}=K(K+1)$ with $\left|S_{1}-S_{2}\right| \leqslant K \leqslant\left(S_{1}+S_{2}\right)$. On these multiplets, $\rho^{T_{2}}$ acts as a constant. As seen above, the eigenvalue corresponding to the largest $K$ multiplet is always non-negative.

\section{THE CASE $S_{2}=1 / 2$}

Let us now consider a system consisting of a spin $\vec{S}_{1}$ of arbitrary length $S$ and a spin $\vec{S}_{2}$ of length $1 / 2$. Here, a general $\mathrm{SU}(2)$-invariant density matrix has the form

$$
\begin{aligned}
\rho= & \frac{F}{2 S} \sum_{J^{z}=-S+1 / 2}^{S-1 / 2}\left|S-\frac{1}{2}, J^{z}\right\rangle_{00}\left|S-\frac{1}{2}, J^{z}\right| \\
& +\frac{1-F}{2 S+2} \sum_{J^{z}=-S-1 / 2}^{S+1 / 2}\left|S+\frac{1}{2}, J^{z}\right\rangle_{00}\left\langle S+\frac{1}{2}, J^{z}\right| .
\end{aligned}
$$

The quantity $F \in[0,1]$ is, in thermal equilibrium, a function of temperature and, in the case of $\rho$ being a reduced density matrix of a larger system, it contains information about the entire system which has been traced out except for the spins $\vec{S}_{1}$ and $\vec{S}_{2}$. By expressing $F$ in terms of the projector onto the $J=S-1 / 2$ multiplet one finds $F=\left(S-2\left\langle\vec{S}_{1} \cdot \vec{S}_{2}\right\rangle\right) /(2 S+1)$, where \langle\rangle denotes the expectation value with respect to $\rho$. Thus, $\rho$ is completely determined by the correlator $\left\langle\vec{S}_{1} \cdot \vec{S}_{2}\right\rangle$.

In order to perform a partial transposition on $\rho$ it is convenient to express it in a basis of tensor product eigenstates $\left|S^{z}, \pm 1 / 2\right\rangle$ of $S_{1}^{z}$ and $S_{2}^{z}$. Using the well-known ClebschGordan coefficients for coupling a spin $S$ to a spin $1 / 2$, the nonvanishing matrix elements are given by

$$
\begin{aligned}
\left\langle S^{z}, \pm 1 / 2|\rho| S^{z}, \pm 1 / 2\right\rangle= & \frac{1}{2 S+1}\left(\frac{\left(S \mp S^{z}\right) F}{2 S}\right. \\
& \left.+\frac{\left(S \pm S^{z}+1\right)(1-F)}{2 S+2}\right),
\end{aligned}
$$

$$
\begin{aligned}
& \left\langle S^{z}, 1 / 2|\rho| S^{z}+1,-1 / 2\right\rangle \\
& \quad=\frac{\sqrt{\left(S-S^{z}\right)\left(S+S^{z}+1\right)}}{2 S+1}\left(-\frac{F}{2 S}+\frac{1-F}{2 S+2}\right) .
\end{aligned}
$$

The partial transpose $\rho^{T_{2}}$ is diagonal on the subspace spanned by $|S,-1 / 2\rangle,|-S,+1 / 2\rangle$ lying in the largest $\vec{K}$ multiplet with the eigenvalue $\lambda_{1}:=\langle S,-1 / 2|\rho| S,-1 / 2\rangle=$ $\langle-S, 1 / 2|\rho|-S, 1 / 2\rangle$, where

$$
\lambda_{1}=\frac{1}{2 S+1}\left(F+\frac{1-F}{2 S+2}\right)
$$

On the remaining Hilbert space, the partial transpose is block diagonal, where the blocks act on subspaces spanned by the basis vectors $\left|S^{z},-1 / 2\right\rangle,\left|S^{z}+1,1 / 2\right\rangle$ and have the form

$$
\left(\begin{array}{cc}
\left\langle S^{z},-1 / 2|\rho| S^{z},-1 / 2\right\rangle & \left\langle S^{z}, 1 / 2|\rho| S^{z}+1,-1 / 2\right\rangle \\
\left\langle S^{z}, 1 / 2|\rho| S^{z}+1,-1 / 2\right\rangle & \left\langle S^{z}+1,1 / 2|\rho| S^{z}+1,1 / 2\right\rangle
\end{array}\right) .
$$

The eigenvalues of these submatrices are given by $\lambda_{1}$ and

$$
\lambda_{2}=\frac{1}{2 S+1}-\frac{1}{2 S} F
$$

These eigenvalues do not depend on $S^{z}$. Therefore, $\lambda_{1}$ and $\lambda_{2}$ occur with the multiplicities $2 S+2$ and $2 S$, respectively, in accordance with the above general results. Moreover, $\lambda_{1}$ is always positive, while $\lambda_{2}$ becomes negative for $F>2 S /(2 S$ +1 ), or, equivalently,

$$
\left\langle\vec{S}_{1} \cdot \vec{S}_{2}\right\rangle<-\frac{S}{2}
$$

Thus, our state has a nonpositive partial transpose if and only if the correlator $\left\langle\vec{S}_{1} \cdot \vec{S}_{2}\right\rangle$ is negative and larger in modulus than $S / 2$. This is the maximum value $\left|\left\langle\vec{S}_{1} \cdot \vec{S}_{2}\right\rangle\right|$ an achieve in a separable state. This intuitive very reasonable criterion includes earlier results by Wang and Zanardi [14] who investigated the case $S_{1}=S_{2}=1 / 2$ by evaluating the entanglement of formation [30] using Wootters' concurrence [31]. Unfortunately, this is not a viable route for $S_{1}>1 / 2$, since Wootters' construction appears to be restricted to the case of two qubits. Moreover, with increasing $S$, the states with a nonpositive partial transpose have increasing weight in the smaller multiplet $J=S-1 / 2$, approaching unity for $S \rightarrow \infty$.

If a given state has a negative partial transpose it is necessarily entangled. Moreover, in the case of SU(2)-invariant states with $S_{2}=1 / 2$ studied in this section, a positive partial transpose, i.e., $\left\langle\vec{S}_{1} \cdot \vec{S}_{2}\right\rangle \geqslant-S / 2$, is also a sufficient criterion for separable states. We prove this fact by explicitly constructing a decomposition consisting of projectors on porduct states. If a given state has a positive partial transpose, we can write $\left\langle\vec{S}_{1} \cdot \vec{S}_{2}\right\rangle=(S / 2) \cos (\gamma)$ with some real angle $\gamma$. Now let $|0\rangle$ denote a spin-coherent state $[32,33]$ of $S_{1}$ pointing in some arbitrary direction and $|\gamma\rangle$ denote a spin-coherent state of $S_{2}$ with its polarization direction forming angle $\gamma$ with the polarization direction of $S_{1}$. In the pure product state $|0\rangle$ 
$\otimes|\gamma\rangle$, we have by construction $\left\langle\vec{S}_{1} \cdot \vec{S}_{2}\right\rangle=(S / 2) \cos (\gamma)$, and the value of this correlator is invariant under all uniform rotations of both spins. Now consider

$$
\begin{aligned}
\rho= & N(S) \int d^{3} \eta\left\{\left[U_{1}(\eta) \otimes U_{2}(\eta)\right](|0\rangle \otimes|\gamma\rangle\langle 0| \otimes\langle\gamma|)\right. \\
& \left.\times\left[U_{1}(\eta) \otimes U_{2}(\eta)\right]^{+}\right\},
\end{aligned}
$$

where the integration goes over all simultaneous rotations parametrized as $U_{a}=\exp \left(i \vec{\eta} \cdot \vec{S}_{a}\right), a \in\{1,2\}$, and $N(S)$ is a normalization constant. Thus, state (9) is a separable state which is obviously invariant under simultaneous rotations of both spins and fulfills $\left\langle\vec{S}_{1} \cdot \vec{S}_{2}\right\rangle=(S / 2) \cos (\gamma)$. Since such an $\mathrm{SU}(2)$-invariant state is uniquely determined by this correlator, we have constructed a decomposition of the original state in terms of projectors on product states, which completes the proof.

Let us now discuss the above result with respect to isotropic Heisenberg lattice-spin models as studied intensively in condensed-matter physics and statistical mechanics. Previous studies have concentrated on one-dimensional systems. This has on one hand the practical reason that for such systems the body of exact results concerning correlations is largest. On the other hand, this is due to the fact that quantum correlations can generically be expected to become weaker with increasing spatial dimension, i.e., with increasing number of neighbors to each spin. Therefore, onedimensional systems are the most attractive to look for equilibrium quantum entanglement.

Since quantum correlations such as $\left\langle\vec{S}_{1} \cdot \vec{S}_{2}\right\rangle$ can generally be expected to decay with increasing temperature, criterion (8) defines implicitly a threshold temperature for the occurrence of a nonpositive partial transpose, provided inequality (8) is fulfilled in the ground state at $T=0$ [14]. This can only be the case in antiferromagnetic or, for $S_{1}>S_{2}=1 / 2$, ferrimagnetic systems. In particular, in an antiferromagnetic spin$1 / 2$ chain correlations are generically of the form

$$
\left\langle\vec{S}_{m} \cdot \vec{S}_{m+n}\right\rangle=(-1)^{n} \chi(n),
$$

where $m$ denotes some lattice site in the translationally invariant chain, $n$ is the number of lattice sites between the spins considered, and $\chi$ is a positive and monotonously decaying function. The alternating sign resembles Néel ordering as it is present in the ground state of a classical antiferromagnet. It follows that the reduced, equilibrium, two-spin density matrix can only be entangled if the spins involved reside on different sublattices (corresponding to odd $n$ ). This intuitively clear finding also holds for generic antiferromagnets or ferrimagnets on bipartite lattices in higher spatial dimension.

The spin-1/2 Heisenberg chain with antiferromagnetic exchange between nearest neighbors is described by the Hamiltonian $\mathcal{H}=\Sigma_{m} \vec{S}_{m} \vec{S}_{m+1}$ Here, the correlator $\left\langle\vec{S}_{m} \cdot \vec{S}_{m+1}\right\rangle$ is equal to the ground-state energy per spin and given by $\ln 2$ $-1 / 4 \approx-0.443<-0.25$ [34]. Thus, criterion (8) is fulfilled [14]. However, for larger distances between the spins, $n$ $\in\{3,5, \ldots\}$, numerical data [35] shows that inequality (8) is violated and, according to the Peres-Horodecki criterion, the corresponding reduced density matrix is separable. The same statements apply to another typical antiferromagnetic spin$1 / 2$ wave function, the so-called Gutzwiller wave function [36], where $\left\langle\vec{S}_{m} \cdot \vec{S}_{m+n}\right\rangle$ can be evaluated analytically for all $n$ [37].

In summary, one-dimensional antiferromagnetic, isotropic, Heisenberg models of spins $1 / 2$ do not generically appear to be in thermal equilibrium a particularly strong source of entanglement, since usually only the reduced density matrices of neigboring spins are inseparable, while all others are nonentangled. This result might appear somewhat surprising since such systems are usually considered to have particularly strong spin correlations because of the small spin length as well as the low spatial dimension [33]. However, as seen here, these strong quantum correlations do not, in general, translate to long-ranged entanglement in equilibrium, reduced density matrices.

As pointed out already, this finding cannot be expected to change in the case of higher spatial dimension. Moreover, ferrimagnetic systems involving spins $S_{1}>1 / 2$ will generically have the same properties, since we have the same type of criterion (8) for the partial transpose being nonpositive. This criterion requires sufficiently strong quantum fluctuations which are generically reduced with increasing spin length. To illustrate these trends let us rewrite criterion (8) in the form $\left\langle\vec{S}_{1} \cdot \vec{S}_{2}\right\rangle /\left(S_{1} S_{2}\right)<-1$. For a ferrimagnetic chain consisting of alternating spins $S_{1}=1, S_{2}=1 / 2$, a numerical estimate for the left-hand side of this inequality in the case of neighboring spins is given by [38] $\left\langle\vec{S}_{1} \cdot \vec{S}_{2}\right\rangle /\left(S_{1} S_{2}\right)=$ -1.455 . For a two-dimensional spin-1/2 antiferromagnet on the square lattice one finds [39] $\left\langle\vec{S}_{1} \cdot \vec{S}_{2}\right\rangle /\left(S_{1} S_{2}\right)=-1.344$. Both values are larger than the result for the spin-1/2 chain as discussed above, $\left\langle\vec{S}_{1} \cdot \vec{S}_{2}\right\rangle /\left(S_{1} S_{2}\right)=-1.773$, indicating the suppression of pairwise entanglement with increasing spatial dimension and lengths of spins involved.

\section{THE CASE $S_{2} \geqslant 1$}

We now turn to the case $S_{1}=: S \geqslant 1, S_{2}=1$. Here, the general $\mathrm{SU}(2)$-invariant density matrix reads

$$
\begin{aligned}
\rho= & \frac{G}{2 S-1} \sum_{J^{z}=-S+1}^{S-1}\left|S-1, J^{z}\right\rangle_{00}\left\langle S-1, J^{z}\right| \\
& +\frac{H}{2 S+1} \sum_{J^{z}=-S}^{S}\left|S, J^{z}\right\rangle_{00}\left\langle S, J^{z}\right| \\
& +\frac{1-G-H}{2 S+3} \sum_{J^{z}=-S-1}^{S+1}\left|S+1, J^{z}\right\rangle_{00}\left\langle S+1, J^{z}\right| .
\end{aligned}
$$

Expressing the quantities $G$ and $H$ in terms of projectors onto the multiplets on the total spin $J \in\{S-1, S, S+1\}$ one finds

$$
G=\frac{1}{S(2 S+1)}\left[-S-(S-1)\left\langle\vec{S}_{1} \cdot \vec{S}_{2}\right\rangle+\left\langle\left(\vec{S}_{1} \cdot \vec{S}_{2}\right)^{2}\right\rangle\right],
$$




$$
H=1-\frac{1}{S(S+1)}\left[\left\langle\vec{S}_{1} \cdot \vec{S}_{2}\right\rangle+\left\langle\left(\vec{S}_{1} \cdot \vec{S}_{2}\right)^{2}\right\rangle\right]
$$

Therefore, $\rho$ is completely determined by the correlators $\left\langle\vec{S}_{1} \cdot \vec{S}_{2}\right\rangle$ and $\left\langle\left(\vec{S}_{1} \cdot \vec{S}_{2}\right)^{2}\right\rangle$ and has three different eigenvalues with degeneracies $2 S-1,2 S+1$, and $2 S+3$, corresponding to multiplets of the total spin $\vec{J}$. The three different eigenvalues of $\rho^{T_{2}}$ can be found in the subspaces spanned by $\left(\mid S^{z}\right.$ $\left.+1,1\rangle,\left|S^{z}, 0\right\rangle,\left|S^{z}-1-1\right\rangle\right),\left|S^{z}\right| \neq S$ having $K^{z}=S^{z}$. With respect to this basis, $\rho^{T_{2}}$ reads

$$
\left(\begin{array}{ccc}
\alpha\left(S^{z}\right) & \delta\left(S^{z}\right) & \varepsilon\left(S^{z}\right) \\
\delta\left(S^{z}\right) & \beta\left(S^{z}\right) & \eta\left(S^{z}\right) \\
\varepsilon\left(S^{z}\right) & \eta\left(S^{z}\right) & \gamma\left(S^{z}\right)
\end{array}\right)
$$

with

$$
\begin{gathered}
\alpha\left(S^{z}\right)=\left\langle S^{z}+1,1|\rho| S^{z}+1,1\right\rangle, \\
\beta\left(S^{z}\right)=\left\langle S^{z}, 0|\rho| S^{z}, 0\right\rangle, \\
\gamma\left(S^{z}\right)=\left\langle S^{z}-1-1|\rho| S^{z}-1,-1\right\rangle, \\
\delta\left(S^{z}\right)=\left\langle S^{z}, 1|\rho| S^{z}+1,0\right\rangle, \\
\varepsilon\left(S^{z}\right)=\left\langle S^{z}+1,-1|\rho| S^{z}-1,1\right\rangle, \\
\eta\left(S^{z}\right)=\left\langle S^{z},-1|\rho| S^{z}-1,0\right\rangle .
\end{gathered}
$$

Unfortunately, the evaluation of the matrix elements in Eq. (14) for general values of $S$ and $S^{z}$ turns out to be extremely tedious because the form of the Clebsch-Gordan coefficients is drastically more complicated than in the previous case $S_{2}=1 / 2$. For simplicity, we therefore concentrate on the case $S=1$ where the only block of form (14) corresponds to $S^{z}$ $=0$ with $\alpha=\gamma$ and $\delta=\eta$. In this case we have the eigenvalues

$$
\begin{gathered}
\mu_{1}=\frac{1}{30}+\frac{3}{10} G+\frac{2}{15} H, \\
\mu_{2}=\frac{1}{6}-\frac{1}{2} G, \\
\mu_{3}=\frac{1}{3}-\frac{2}{3} H .
\end{gathered}
$$

The eigenvalue $\mu_{1}$ is always positive and corresponds to the largest $K$ multiplet. Thus, $\rho^{T_{2}}$ has negative eigenvalues if and only if $G>1 / 3$ or $H>1 / 2$. Expressed in terms of correlators, these conditions read

$$
\begin{gathered}
2<\left\langle\left(\vec{S}_{1} \cdot \vec{S}_{2}\right)^{2}\right\rangle, \\
1>\left\langle\vec{S}_{1} \cdot \vec{S}_{2}\right\rangle+\left\langle\left(\vec{S}_{1} \vec{S}_{2}\right)^{2}\right\rangle .
\end{gathered}
$$

Similarly as in the previous case $S_{1}=S, S_{2}=1 / 2$, the twospin density matrix has a nonpositive partial transpose only if the weight of the smaller multiplets is sufficiently large. We note that, different from the case $S_{1}=S, S_{2}=1 / 2$, an analogous proof for the sufficiency of the Peres-Horodecki criterion cannot be given for $S_{2} \geqslant 1$ since such states are determined by more than just a single correlator.

\section{CONCLUSIONS}

We have analyzed the pairwise quantum entanglement in SU(2)-invariant quantum spin systems by studying their behavior under partial transposition. As a general result, the partial transpose of an SU(2)-invariant two-spin state has the same multiplet structure and degeneracies as the original matrix with eigenvalue of largest multiplicity being nonnegative. SU(2)-invariant density matrices arise from thermal equilibrium states of isotropic-spin systems in sufficiently low spatial dimension. The case $S_{1}=S, S_{2}=1 / 2$ can be solved completely and is discussed in detail with respect to the well-known Heisenberg spin models. Moreover, in this case the Peres-Horodecki ciriterion turns out to be a sufficient condition for nonseparability. As a general trend, low spatial dimension and small lengths of the spins involved tend to facilitate the occurrence of inseparable equilibrium states. However, inseparability occurs typically only between neighboring spins in such spin-lattice systems. In this sense, isotropic Heisenberg spin models do not appear to be a particularly strong source of quantum entanglement, at least as far as their equilibrium properties are concerned. Finally, we have also characterized the properties of SU(2)invariant states of two spins of length 1 under partial transposition.

\section{ACKNOWLEDGMENTS}

I thank Maciej Lewenstein and R.-F. Werner for useful discussions.
[1] A. Einstein, B. Podolsky, and N. Rosen, Phys. Rev. 47, 777 (1935).

[2] E. Schrödinger, Naturwissenschaften 23, 807 (1935).

[3] For a general overview see, e.g., M.A. Nielsen and I.L. Chuang, Quantum Computation and Quantum Information (Cambridge University Press, Cambridge, 2000); for recent reviews on quantum entanglement see Refs. [4-6]

[4] M. Lewenstein, D. Bruß, J.I. Cirac, M. Kus, J. Samsonowicz, A. Sanpera, and R. Tarrach, J. Mod. Opt. 47, 2841 (2000).

[5] B.M. Terhal, Theor. Comput. Sci. 287, 313 (2002).
[6] D. Bruß, J. Math. Phys. 43, 4237 (2002).

[7] W.K. Wootters, e-print quant-ph/0001114.

[8] K.M. O'Connor and W.K. Wootters, Phys. Rev. A 63, 052302 (2001).

[9] M.C. Arnesen, S. Bose, and V. Vedral, Phys. Rev. Lett. 87, 017901 (2001).

[10] D. Gunlycke, V.M. Kendon, V. Vedral, and S. Bose, Phys. Rev. A 64, 042302 (2001).

[11] X. Wang, H. Fu, and A.I. Solomon, J. Phys. A 34, 11307 (2001). 
[12] X. Wang, New J. Phys. 4, 11 (2002); X. Wang, Phys. Rev. A 66, 034302 (2002).

[13] X. Wang, Phys. Rev. A 66, 044305 (2002).

[14] X. Wang and P. Zanardi, Phys. Lett. A 301, 1 (2002).

[15] G.L. Kamta and A.F. Starace, Phys. Rev. Lett. 88, 107901 (2002).

[16] A. Osterloh, L. Amico, G. Falci, and R. Fazio, Nature (London) 416, 608 (2002).

[17] T.J. Osborne and M.A. Nielsen, Phys. Rev. A 66, 032110 (2002).

[18] I. Bose and E. Chattopadhyay, Phys. Rev. A 66, 062320 (2002).

[19] D.A. Meyer and N.R. Wallach, J. Math. Phys. 43, 4273 (2002).

[20] G. Vidal, J.I. Latorre, E. Rico, and A. Kitaev, Phys. Rev. Lett. 90, 227902 (2003).

[21] J.I. Latorre, E. Rico, and G. Vidal, e-print quant-ph/0304098.

[22] B.-Q. Jin and V.E. Korepin, quant-ph/0304108.

[23] We note that this restriction to the density matrix excludes spontaneous breaking of the rotational symmetry as it occurs in ferromagnetic or ferrimagnetic phases. However, such symmetry breaking is not possible at finite temperature in systems with a spatial dimension not larger than 2, N.D. Mermin and H. Wagner, Phys. Rev. Lett. 17, 1133 (1966), and systems of this kind are in the focus of previous and present investigations.

[24] A. Peres, Phys. Rev. Lett. 77, 1413 (1996).
[25] M. Horodecki, P. Horodecki, and R. Horodecki, Phys. Lett. A 223, 8 (1996).

[26] P. Horodecki, Phys. Lett. A 232, 333 (1997).

[27] K.G.H. Vollbrecht and R.F. Werner, Phys. Rev. A 64, 062307 (2002).

[28] After submitting an earlier version of this paper, I learned about related unpublished work by B. Hendriks and R.F. Werner [B. Hendriks, Diploma thesis, University of Braunschweig, Germany, 2002].

[29] As it was noticed in Ref. [27], there is a slight ambiguity in the construction of $U_{1} \otimes U_{2}^{*}$ concerning a global phase. This phase, however, does not influence the action of $U_{1} \otimes U_{2}^{*}$ on any operator.

[30] C.H. Bennett, D.P. DiVincenzo, J.A. Smolin, and W.K. Wootters, Phys. Rev. A 54, 3824 (1996).

[31] W.K. Wootters, Phys. Rev. Lett. 80, 2245 (1998).

[32] J.M. Radcliffe, J. Phys. A 4, 313 (1971).

[33] See, e.g., A. Auerbach, Interacting Electrons and Quantum Magnetism (Springer, New York, 1994).

[34] H. Bethe, Z. Phys. 71, 205 (1931); L. Hulthén, Ark. Mat., Astron. Fys. 26A, 1 (1938).

[35] M. Betsuyaku and T. Yokota, Phys. Rev. B 33, 6505 (1986).

[36] M.C. Gutzwiller, Phys. Rev. Lett. 10, 159 (1963).

[37] W. Metzner and D. Vollhardt, Phys. Rev. Lett. 59, 121 (1987); F. Gebhard and D. Vollhardt, ibid. 59, 1472 (1987).

[38] S. Brehmer, H.-J. Mikeska, and S. Yamamoto, J. Phys.: Condens. Matter 9, 3921 (1997).

[39] T. Barnes and E.S. Swanson, Phys. Rev. B 37, 9405 (1988). 